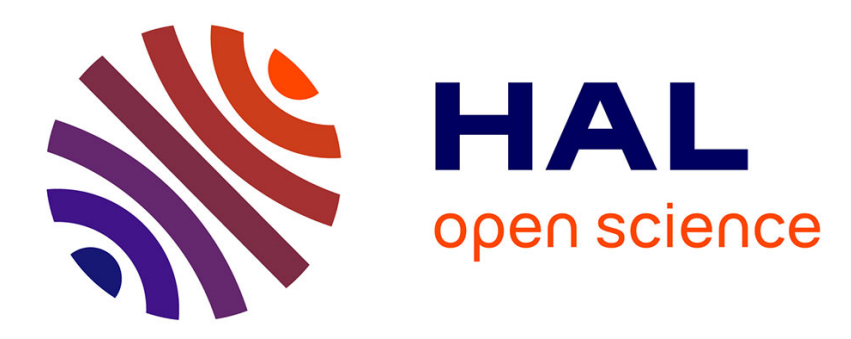

\title{
MÉTHODE SIMPLE ET FACILE POUR LA DÉTERMINATION DE LA MATIÈRE GRASSE DANS LE LAIT
}

Secondo Repetto

\section{To cite this version:}

Secondo Repetto. MÉTHODE SIMPLE ET FACILE POUR LA DÉTERMINATION DE LA MATIÈRE GRASSE DANS LE LAIT. Le Lait, 1935, 15 (141), pp.15-19. hal-00895181

\section{HAL Id: hal-00895181 https://hal.science/hal-00895181}

Submitted on 1 Jan 1935

HAL is a multi-disciplinary open access archive for the deposit and dissemination of scientific research documents, whether they are published or not. The documents may come from teaching and research institutions in France or abroad, or from public or private research centers.
L'archive ouverte pluridisciplinaire HAL, est destinée au dépôt et à la diffusion de documents scientifiques de niveau recherche, publiés ou non, émanant des établissements d'enseignement et de recherche français ou étrangers, des laboratoires publics ou privés. 
Le troisième (élimination de produits indésirables formés par certains microbes) est le même que celui que nous avons rencontré à propos de l'élimination des mauvaises saveurs et odeurs provenant de l'alimentation des vaches : ici comme là, il consiste à aérer le lait après pasteurisation, c'est-à-dire à pratiquer la réfrigération à l'air libre.

Nous tombons ici sur un remède commun à deux causes différentes et c'est fort heureux car les conclusions pratiques générales en seront bien simplifiées.

(A suivre.)

\section{MÉTHODE SIMPLE ET FACILE POUR LA DÉTERMINATION DE LA MATIËRE GRASSE DANS LE LAIT}

\section{par le Docteur Secondo REPETTO}

Istituto di Zootecnia e Igiene Zooteoniea del R. Istituto Superiore di Medicina Veterinaria di Sassari (Directeur : Professeur A. Campus).

Au cours de mes expériences de détermination de la matière grasse dans le lait de brebis, selon les méthodes Gottlieb-Röse, Gerber, Neusal, Cavalli, Maccagno, Höyberg, Morsin, Magliano et Singh - dont j'ai exposé les résultats dans un ouvrage précédent (1) -, j'ai eu l'oecasion d'observer que quelques-unes des méthodes susdites, pour lesquelles on se sert de solutions alcalines, en gros se ressemblent.

Dans mes nombreuses expériences d'orientation, j'ai pu constater que ces méthodes étaient fondées sur l'action combinée de l'hy drate de sodium, indispensable pour dissoudre et rendre fluides les substances protéiques, avec l'alcool isobutylique nécessaire pour faire affleurer et provoquer la séparation de la matière grasse et permettre la formation d'un dépôt limpide.

Les autres substances qui entrent dans la composition du réactif - non toutes encore bien connues, les Maisons fabricantes aimant les tenir secrètes - doivent avoir une action secondaire.

En me servant des butyromètres communs Gerber, Morsin et Höyberg, et, en partant d'une quantité de lait déterminée, j'ajoutais, en des proportions variables, la soude et l'alcool isobutylique. J'ai pu ainsi constater que, en quelques-uns des butyromètres dont je me servais, des petites gouttes de matière grasse se séparaient, pareillement à ce qui arrivait lorsque je suivais les méthodes susdites, et ces gouttes affleuraient à la surface, formant une couche plus ou moins continue.

(1) Considérations et remarques sur quelques méthodes pour la détermination de la graisse dans le lait de brebis. L'Italia Agricola, 1934. 
Si la quantité d'aleool isobutylique était trop petite, ou trop forte la concentration de la solution de soude, la matière grasse ne devenait pas limpide, ou, du moins, elle mettait un temps assez long avant d'affleurer. Une séparation rapide de la matière grasse se vérifiait au contraire quand la solution, au-dessous, paraissait limpide et un coagulum se déposait au fond.

J'ai alors essayé, toujours à la suite d'essais d'orientation et en me servant toujours des susdits butyromètres, d'établir quelles étaient les conditions les meilleures pour obtenir une séparation facile et un rapide affleurement de la matière grasse. Après de très nombreuses épreuves concernant ce dernier temps, je suis arrivé à des résultats assez satisfaisants, en employant la quantité de réactif et en suivant la technique que je vais exposer.

Butyromètres Gerber. - Lorsque je me suis servi des butyromètres Gerber, j'ai employé :

Solution de $\mathrm{NaOH}$ au $6,5 \%$;

Alcool isobutylique ;

et, comme matériel : un bain-marie, un centrifugeur, une pipette de $10 \mathrm{cc}$. pour le lait, une pipette de $10,5 \mathrm{cc}$. pour la soude, une de $1 \mathrm{cc}$. pour l'alcool isobutylique, des butyromètres Gerber, des bouchons Fibu, un thermomètre.

A) Et voilà les quantités des liquides que j'ai employés :

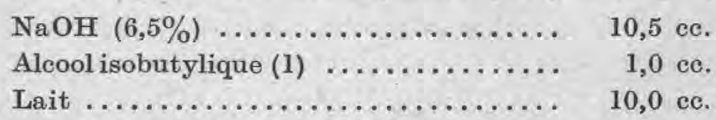

On agite le butyromètre énergiquement pendant quelque 30 secondes, on le renverse quatre ou cinq fois, dans le but d'obtenir une solution homogène ; on le plonge dans un bain d'eau à $60^{\circ}-62^{\circ} \mathrm{C}$. pendant 8 minutes; on l'enlève du bain et on l'agite encore énergiquement, ayant soin de tenir le butyromètre horizontalement, jusqu'à ce que les grumeaux se soient complètement dissous (il faut agiter de 20 à 30 secondes environ). On renverse encore le butyrumètre 4 à 5 fois, on le remet dans le bain pendant 3 à 5 minutes, et, enfin, on centrifuge pendant 4 à 5 minutes. On fait la lecture, qui donne directement en \% la quantité de matière grasse, après avoir remis pour quelques instants le butyromètre dans le bain à $60^{\circ} \mathrm{C}$.

B) Tout en laissant la technique invariée, j'ai inverti les quantités de solution de soude et de lait, c'est-à-dire que j'ai pris :

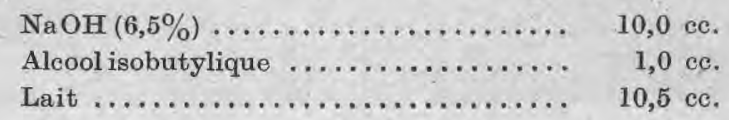

(1) Mesurer avec la plus grande préeision la quantité d'alcool. 
Butyromètres Morsin. - Lorsque j'ai fait usage des butyromètres Morsin et Höyberg, j'ai employé :

Solution de $\mathrm{NaOH} 5,0 \%$;

Alcool isobutylique ;

et comme matériel : un bain-marie, une pipette de $5,5 \mathrm{cc}$. pour la soude, une de 9,5 ec. pour le lait, une de 1,0 ce. pour l'alcool isobutylique, des bouchons Fibu ou Höyberg, un thermomètre.

Exícution. - On verse dans le butyromètre :

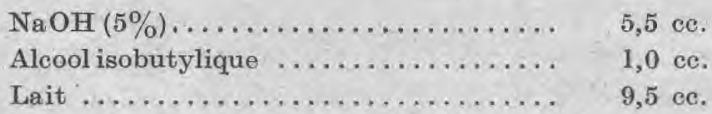

On peut suivre les deux procédés suivants :

๙) On agite énergiquement le butyromètre pendant environ 30 secondes et le renverse 2 à 3 fois, pour rendre le mélange homogène; on le met dans un bain de $60^{\circ}$ à $62^{\circ} \mathrm{C}$. pendant 7 à 8 minutes ; on l'enlève du bain; on agite énergiquement, en tenant le butyromètre horizontalement, jusqu'à ce que les grumeaux du coagulum soient complètement dissous (il suffit pour cela de 20 à 30 secondes) ; on le renverse 2-3 fois; on le remet dans le bain, et, après 10-15 minutes, on fait la lecture. L'usage du centrifugeur reste ainsi superflu.

ß) On suit la technique précédemment citée pour les butyromètres Gerber.

Par les procédés déjà exposés, la matière grasse se sépare en de petites gouttes qui montent rapidement à la surface et le liquide restant au-dessous devient très limpide, couleur orange, tandis que les flocons de coagulum se déposent au fond.

J'ai toujours eu soin d'employer, soit pour le lait, soit pour la solution de soude, et surtout pour l'alcool isobutylique, des quantités de réactif et de lait dont il était possible d'exprimer les valeurs en nombres entiers.

\section{Précision de la méthode.}

Des essais de comparaison que nous avons faits à l'égard de la méthode Gerber, il est résulté que, en suivant le procédé A) (c'est-à-dire en utilisant des butyromètres communs Gerber et en employant $10,0 \mathrm{ec}$. de lait), les variations n'ont pas été supérieures à $\pm 0,15$ et la moyenne des différences a été de $+0,001$. En employant, au contraire, 10,5 cc. de lait (procédé B), la moyenno des différences a été de $+0,14$.

Se servant, au contraire, des butyromètres Morsin (on devrait obtenir les mêmes données même avec les butyromètres Höyberg, qui ont la même capacité et la même graduation que les Morsin), 
la moyenne des différences en \pm , toujours par rapport à la méthode Gerber, a été de - 0,002 pour les épreuves dans lesquelles on a suivi la technique présentée en $\alpha$ ) et de $+0,14$ pour celle présentée en $\beta$ ).

Outre ces déterminations exécutées avec du lait de vache, nous en avons fait quelques-unes, nous servant toujours des butyromètres Morsin, avec du lait de brebis, dilué à $50 \%$, lés contrôlant soit par la méthode Gerher, soit par la méthode pondérale RöseGottlieh.

En suivant la technique indiquée en $\alpha$ ), c'est-à-dire ne nous servant pas du centrifugeur, la variation moyenne a été, par rapport à la méthode Gottlieb-Röse, de $-0,16$, tandis que, en suivant la technique indiquée en $\beta$ ), la variation moyenne n'a été que de $-0,06$.

Les mêmes échantillons de lait avaient donné une différence par rapport à la méthode Gerber, respectivement de $+0,04$ et de $+0,14$.

La technique et la quantité de réactif et de lait indiquées respectivement en $\mathrm{A}$ ) et en $\alpha$ ) nous fournissent des données correspondant à la méthode volumétrique de Gerber, qui est considérée en Italie comme la méthode officielle. Le procédé indiqué en B) et en $\beta$ ) nous fournit au contraire des données très rapprochées de la méthode pondérale Röse-Gottlieb, considérée comme la méthode la plus précise.

Etant donné le nombre limité des expériences de contrôle que nous avons exécutées, nous désirerions que des expérimentateurs appartenant à d'autres Instituts éprouvassent cette méthode et contrôlassent sa précision.

Nous pensons que, de toutes les méthodes qui ont été proposées en ees dernières années pour la détermination de la matière grasse, celle que nous avons indiquée n'est pas inférieure aux autres, et qu'elle pourrait être employée utilement dans la pratique ordinaire.

En effet, cette méthode a pour elle, en dehors de sa simplicité et de la facilité de sa technique, la possibilité de permettre l'exécution, en série, de déterminations différentes, la dépense relativement faible occasionnée par l'achat, d'ailleurs facile, du matériel nécessaire.

Le temps nécessaire pour l'exécution d'une détermination, variant entre 20 et 25 minutes, n'est done pas certainement supérieur au temps nécessaire, à l'exception de la méthode Gerber, aux autres méthodes volumétriques employées ordinairement pour la détermination de la matière grasse.

Le coût de chaque détermination exécutée par cette méthode, en ealeulant le prix de l'alcool isobutylique à 24 lires (1) le litre et celui

(1) La lire vaut environ $1 \mathrm{fr} .30$. 
de L'hydrate de sodium à 16 lires le kilo, serait de 3,6 centimes en utilisant des butyromètres Gerber et de 3 centimes seulement en se servant des butyromètres Morsin ou Höyberg. Ce prix est nettement inférieur à celui de 14-17 centimes qui est admis ordinairement pour les méthodes Gerber, Magliano, Höyberg et Neusal, à celui de 18-20 centimes de la méthode Sing, à celui de 20-30 centimes des méthodes Morsin et Cavalli.

\title{
ESSAIS SUR LA FABRICATION DU PORT-SALUT EN PARTANT DE LAITS PASTEURISÉS REEENEMENCÉS DE LEVAIN LACTIQUE
}

\author{
par CH. PORCHER † et G. THIEULIN
}

Les laits que l'on désire pasteuriser pour la fabrication du Port-Salut donnent parfois des fromages présentant d'assez nombreux trous, ce qui traduit la présence d'une quantité insolite de $B$. lactis aerogenes. Sans doute, le développement de ces derniers est-il facilité par une acidité moindre du lait, tenant vraisemblablement à des laits de mammites. Sur des laits d'acidité normale dont l'acidification part franchement, les ferments lactiques ordinaires font la police du milieu et, dans une certaine mesure, s'opposent au développement exagéré du $B$. lactis aerogenes. Mais, sur des laits malades, alcalins, dont l'acidification tarde, on est conduit à admettre que le $B$. lactis aerogenes, toujours présent dans ces laits, en même temps que le $B$. coli, trouve des conditions plus favorables pour son développement.

Il faut done d'abord s'assurer de la qualité des laits au point de vue de leur réaction et le mieux qu'on puisse faire ici pour ces laits destinés à la fabrication des fromages, e'est d'utiliser le réactif à l'alizarol. Rien n'empêche également de recourir à des catalasimètres plus ou moins rapides, mais, alors que l'épreuve à l'alizarol doit être réalisée sur les laits de tous les fournisseurs, l'épreuve catalasimétrique peut être plus espacée.

La quantité de présure utilisée est à peu près trois fois celle qui devrait être nécessaire pour un emprésurage normal, s'effectuant sur des laits de réaction également normale. Sans doute, en augmentant l'action de la présure, pense-t-on faciliter le caillage ; mais, à la réflexion, c'est l'acidité du lait au départ qu'il faut penser à améliorer. Sur des laits normaux, cette acidité, d'habitude, se déclanche bien et il suffit, pour la fabrication du Port-Salut, que l'acidité acquise ait atteint $2^{\circ}$ ou $3^{\circ}$, e'est-à-dire que l'acidité totale, en degrés Dornic, du lait, soit de 20 à $21^{\circ}$, pour avoir un emprésurage normal et une acidification qui parte bien. Mais, avec des laits alcalins, 